\title{
Femtosecond Laser Induced Periodic Surface Structures on ZnO Thin Films
}

\author{
Marian ZAMFIRESCU*, Magda ULMEANU, Florin JIPA, Octavian CRETU, Antoniu MOLDOVAN, George EPURESCU, \\ Maria DINESCU, Razvan DABU \\ ${ }^{* 1}$ National Institute for Laser Plasma and Radiation Physics, Atomistilor 409, 077125 Magurele, \\ Bucharest, Romania \\ E-mail: marian.zamfirescu@inflpr.ro
}

\begin{abstract}
Laser Induced Periodic Surface Structures (LIPSS) were obtained on $\mathrm{ZnO}$ thin films deposited on sapphire substrate. The structures were creaded by scanning the sample surface with a focalised femtosecond laser beam in air. The period and depth of the structures were measured by Atomic Force Microscopy (AFM) and Scanning Electron Microscopy (SEM). The dependence of the morphology of the periodic structures on the experimental conditions such as laser fluence, laser polarisation and laser scanning speed, were investigated. Ripples with $150 \mathrm{~nm}$ period, spaced by grooves with $50 \mathrm{~nm}$ width and about $100 \mathrm{~nm}$ depth have been obtained on large area by multiple scanning of the sample surface. Such microprocessing method suggests a possible technique to produce nanogratings, micropolarisers, or nanopatterned surfaces for micro-sensors.

DOI: $10.2961 / \mathrm{jlmn} .2009 .01 .0002$
\end{abstract}

Keywords: femtosecond laser processing, ripple, nanogratings, $\mathrm{ZnO}$

\section{Introduction}

The periodic self organized microstructures, commonly known as ripples, has been observed on laser irradiated surfaces even from the beginning times of laser processing of materials [1]. When nanosecond polarised lasers were used, the period $\Lambda$ of laser generated patterns was in the range of few microns, indicating a direct dependence of the periodicity with the laser wavelength. The intensive studies of ripples formation on dielectrics, semiconductors and metals had shown also a strong dependence of the ripples morphology with materials properties such as the refractive index [2-3]. The first explanations of ripple formation were related to the interference of the incident laser beam with scattered or excited surface waves [4-5].

In order to explain the small differences between the experimental data of the period $\Lambda$ and the values of the wavelength in material $\lambda$, the inclination of the sample was also considered, giving the dependence $\Lambda=\lambda /(1 \pm \sin \theta)$, where $\theta$ is the angle between the laser beam and the normal to the surface [6]. This correction was used also to explain the small differences reported in the case of a tilted surface of a deep laser ablated crater.

Once the ultrafast lasers became a very spread tool for micromachining, more puzzling information have been added to the phenomenology of the ripples formation. In contrast to the nanosecond lasers, femtosecond pulses induce sub-wavelength periodic structures, much lower than the values predicted by the classical model. A lot of experimental parameters has been revealed to have influence upon the periodicity of the laser induced surface structures, such as material refractive index, laser fluence, number of laser pulses, pulse duration, laser wavelength, radiation polarisation, incidence angle. As a general observed behavior, the ripples are oriented perpendicular to the polarisation of the radiation, the period decrease with an increasing number of laser pulses, and increase with increasing laser energy [7-15]. Sometimes, some materials are reported to behave very different from the general tendency, this behavior making more difficult to hypothesize a simple and general theoretical model.

In the case of laser micromaching, the formation of the ripples is considered as a drawback in the fabrication of microstructures due to the limited smoothness of the processed surfaces. However, controlling the morphology of the ripples may lead to applications in large-area nanostructuring of materials [16-18].

In this work we study the ripples formation on zinc oxide thin films. $\mathrm{ZnO}$ is an environment-friendly material, suitable for optical devices such as solar cells, efficient blue light emitters, or even polariton lasing devices [17-19]. When a $\mathrm{ZnO}$ film is irradiated by femtosecond laser above the ablation threshold, ripples are created on the surface [22-24]. We studied their dependence on the experimental conditions such as laser fluence, laser polarisation and laser scanning speed.

\section{Experimental}

The equipment used to create the periodic ripples is a standard laser micro-processing setup in air. The laser source is a commercial Ti:Sapphire regenerative amplifier working in chirped pulse amplification (CPA) configuration. The laser delivers pulses with 200 fs duration, at 775 $\mathrm{nm}$ wavelength, and $2 \mathrm{kHz}$ repetition rate. The laser beam is focused to an estimated spot diameter of about $3 \mu \mathrm{m}$ by a NIR microscope objective with 0.5 numerical aperture, $100 \mathrm{X}$ magnification and $12 \mathrm{~mm}$ working distance. The samples are precisely translated and positioned by a XYZ computer controlled translation stage, equipped with stepper motors which provide displacement accuracy in the range of few hundreds of nanometers with maximum translation speed of $2 \mathrm{~mm} / \mathrm{s}$. The processed surface is monitored 
in real-time by a monochrome CCD camera with a $200 \mathrm{~mm}$ tube lens. The optical resolution is below $1 \mu \mathrm{m}$.

The laser pulses are continuously attenuated in the range of few $\mathrm{nJ}$ up to hundreds $\mathrm{nJ}$ by a variable attenuator composed by a half waveplate and a Glan-laser polariser. The laser energy is measured in the front of attenuation system by a laser energy meter and corrected by the optical transmission of the microscope objective. The irradiated samples are un-doped $\mathrm{ZnO}$ thin films (about $150 \mathrm{~nm}$ thickness) deposited on r-cut sapphire (1 $\overline{1} 02)$ substrates by RF plasma assisted pulse laser deposition (PLD). The laser fluence was varied from the laser surface modification threshold of our $\mathrm{ZnO}$ film $-0.25 \mathrm{~J} / \mathrm{cm}^{2}$, up to $0.7 \mathrm{~J} / \mathrm{cm}^{2}$, and maintained below the ablation threshold of sapphire substrate in order to have a selective ablation process. The modification threshold is defined as the lowest laser fluence for which any surface modification can be measured after the laser irradiation.

The surface of the samples was scanned by laser at normal incidence with scanning speed from 0.005 to 1 $\mathrm{mm} / \mathrm{s}$. The scanning direction was in $\mathrm{X}, \mathrm{Y}$ or $\mathrm{Z}$, while the polarisation direction of the electric field was kept fixed. After laser irradiation, the samples are investigated by scanning electron microscopy (SEM) and atomic force microscopy (AFM). The AFM measurements are performed in wave-mode with a cantilever with less than $10 \mathrm{~nm}$ radius of the tip.

\section{Results and discussions}

When the laser fluence is kept near the ablation threshold, by scanning the surface of the $\mathrm{ZnO}$ film in $\mathrm{X}, \mathrm{Y}$ or $\mathrm{Z}$ direction, the SEM images reveal LIPSS with about 150 $\mathrm{nm}$ spacing, much below the laser wavelength.

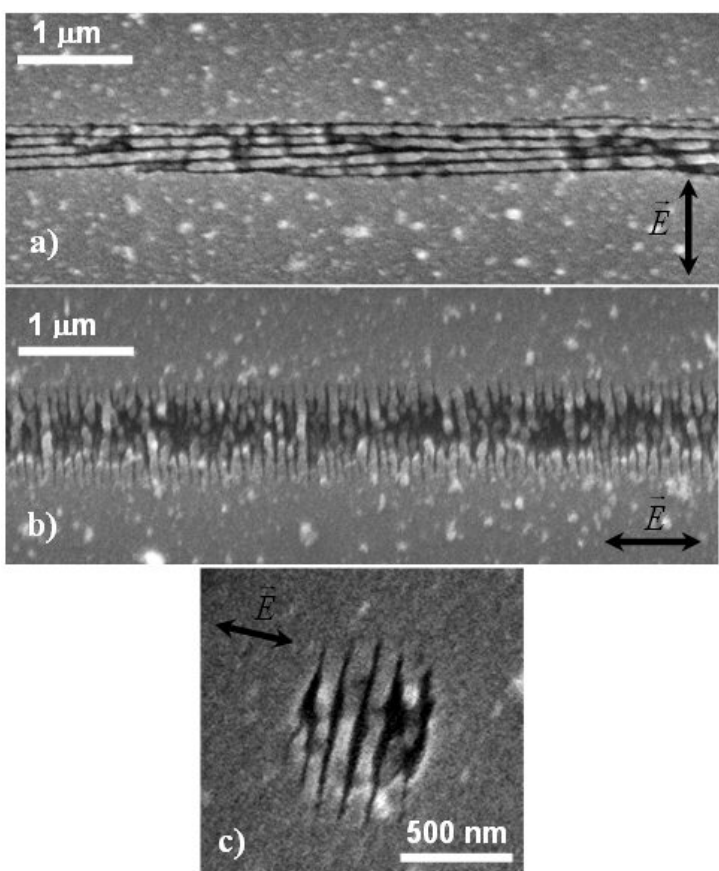

Fig. 1 Ripples on $\mathrm{ZnO}$ film oriented perpendicular to the direction of the electric field. The scanning of the sample is in a) $\mathrm{X}$ direction b) $\mathrm{Y}$ direction and c) $\mathrm{Z}$ direction. The laser fluence was $0.45 \mathrm{~J} / \mathrm{cm}^{2}$ and the scanning speed was $0.01 \mathrm{~mm} / \mathrm{s}$ for each scanning direction.
For the structures presented in the figure 1 the laser fluence was $0.45 \mathrm{~J} / \mathrm{cm}^{2}$ and the scanning speed was $0.01 \mathrm{~mm} / \mathrm{s}$. The periodic induced structures are always oriented perpendicular to the laser polarisation. In the case of the scaning in X direction (figure 1a) only few bifurcations appear, the ripples remaining uninterrupted along many micrometers, separated by clear and uniform grooves with about 50 nm width.

At the same laser energy and scanning speed we moved the sample in $\mathrm{Z}$ direction. Since the Rayleigh length of the focused beam is less than $1 \mu \mathrm{m}$, when a displacement of the sample with $\pm 1 \mu \mathrm{m}$ around the focus plane of the microscope objective is considered, the surface modification effect is rapidly lost. Thus, for a speed of $0.01 \mathrm{~mm} / \mathrm{s}$ and 2 $\mathrm{kHz}$ laser repetition rate, less than 400 laser pulses will contribute to the surface modification process. In these conditions structured dots were created on the sample surfaces (figure 1c). The diameter of the dots is $600 \mathrm{~nm}$ and the step of the periodic structure is $150 \mathrm{~nm}$.

In order to determine the influence of the experimental parameter on the morphology of the LIPSS, a network of parallel lines at different laser energy and scanning speed was realized on the sample surface. We used laser pulses with energy from $18 \mathrm{~nJ}$ to $50 \mathrm{~nJ}$, respectively from 0.25 $\mathrm{J} / \mathrm{cm}^{2}$ up to $0.7 \mathrm{~J} / \mathrm{cm}^{2}$ laser fluences, and scanning speeds from 0.005 to $1 \mathrm{~mm} / \mathrm{s}$. For the threshold laser energy, respectively at laser fluence of $0.28 \mathrm{~J} / \mathrm{cm}^{2}$, no clear periodic structures can be observed; only randomly dispersed grains are formed along the scanning direction for all scanning speeds. At this level of energy the surface starts to be modified, but seem that the laser energy is not high enough to induce self organized periodic structure.

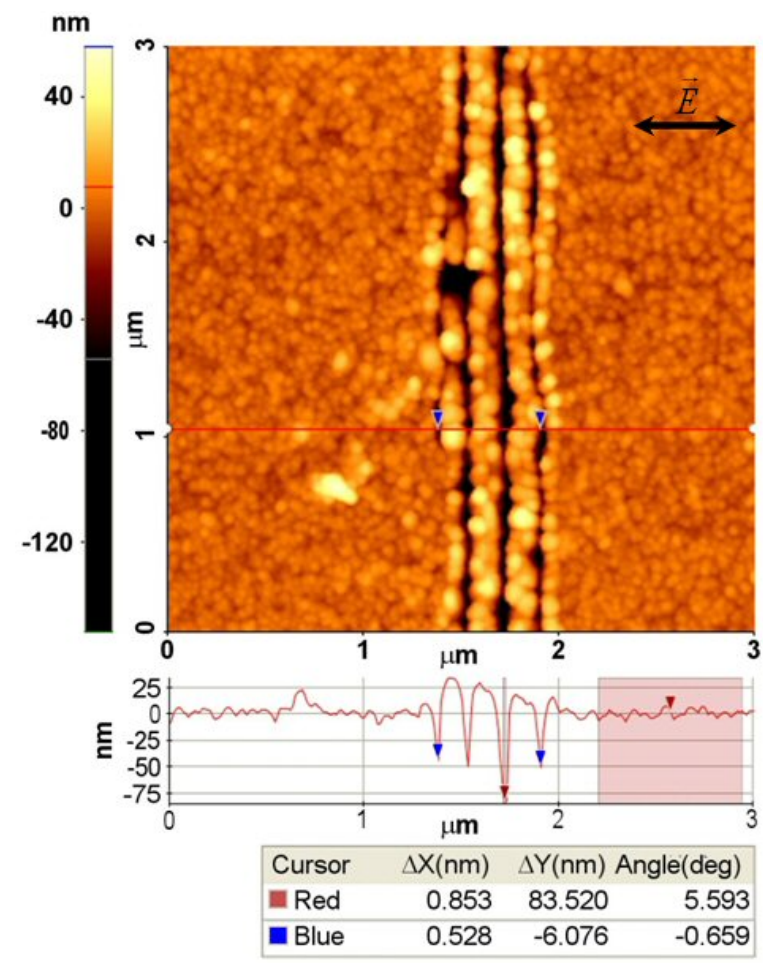

Fig. 2 AFM characterization of ripples on $\mathrm{ZnO}$ films. Laser fluence is $0.45 \mathrm{~J} / \mathrm{cm}^{2}$, and scanning speed is $0.1 \mathrm{~mm} / \mathrm{s}$. The scanning direction was oriented perpendicular to the polarisation. 
At high fluences over $0.65 \mathrm{~J} / \mathrm{cm}^{2}$ the film is completely ablated down to the substrate, then no ripples remain in the irradiated area. A large groove with almost rectangular profile is created. Since the sapphire substrate has a higher ablation threshold, a quite selective ablation process can be expected. Therefore, the thickness of the $\mathrm{ZnO}$ film can be estimated from AFM measurements to be about $150 \mathrm{~nm}$.

For the intermediate laser fluences, periodic structures can be observed. For a scanning speed of $0.1 \mathrm{~mm} / \mathrm{s}$ and laser fluences of $0.34 \mathrm{~J} / \mathrm{cm}^{2}, 0.45 \mathrm{~J} / \mathrm{cm}^{2}$ and $0.57 \mathrm{~J} / \mathrm{cm}^{2}$ we obtained ripples with period of $170 \mathrm{~nm}, 179 \mathrm{~nm}$ and respectively $197 \mathrm{~nm}$. This are averaged values over the periods of all ripples obtained in the same scanning conditions. As it can be observed in the AFM profiles from figure 2, the distance between two adjacent grooves can slightly decrease toward the edge of the laser spot, following the profile of the laser irradiance.

As a general tendency we observed an increasing of the ripples period with the increase of the laser fluence. Thus, the LIPSS dependence with the pulse energy is in good agreement with previous results $[7,8]$.

The same dependence for the depth of the grooves we observed. These become deeper when the laser energy increases. At $0.57 \mathrm{~J} / \mathrm{cm}^{2}$ fluence and speed $0.1 \mathrm{~mm} / \mathrm{s}$ the depth of the ripples is almost comparable with the film thickness denoting a complete perforation of the $\mathrm{ZnO}$ film down to the substrate. Also at lower energies the real depth of the ripples could be higher than the measured one, a small difference being expected due to the finite radius of the AFM tip.

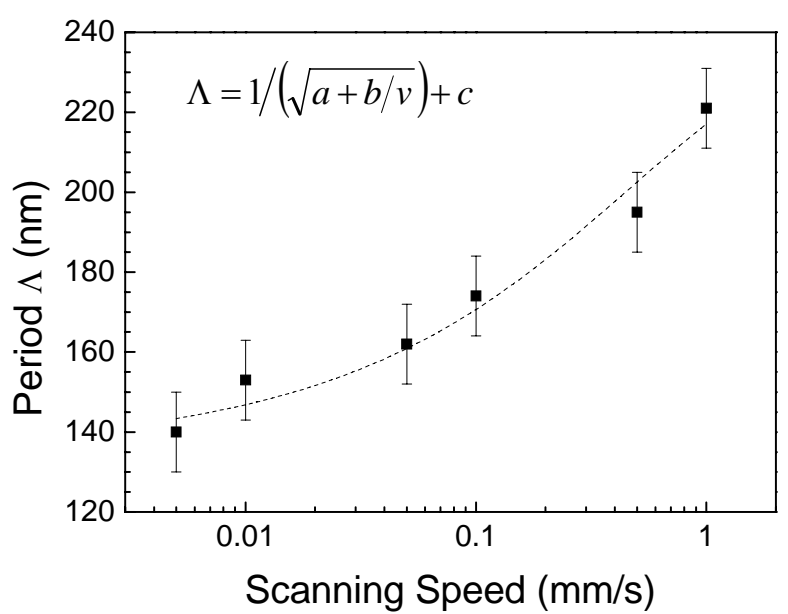

Fig. 3 Dependence of the ripple's period with the scanning speed (log scale) at laser fluence $34 \mathrm{~nJ} / \mathrm{cm}^{2}$. The dotted curve is given by the fitting function shown in the inset.

The figure 3 shows the dependence of the structures period with the scanning speed. The spacing between the structures increases with the increasing of speed, or in other words it increases with decreasing the number of overlapped pulses, as commonly observed $[8,16]$. In our case we can estimate the number of overlapped pulses as following. The diameter of the focused beam is about $3 \mu \mathrm{m}$, nevertheless, we have to take into account the size given by the laser irradiance threshold which induces the surface modification. From the experimental observation this size can be considered to be about $500 \mathrm{~nm}$. Then, for a scanning speed of $0.1 \mathrm{~mm} / \mathrm{s}$ we estimate a number of only 10 overlapped pulses, with about $50 \mathrm{~nm}$ distance between two pulses. The experimental data are fitted by a function $\Lambda=1 /(\sqrt{a+b / v})+c$, where $\Lambda$ is the ripples period, $v$ is scanning speed and $a, b$ and $c$ are the fitting parameters.

When the material is irradiated by a focused femtosecond laser beam, multiphoton absorption and photoionizations occurs. Thus, the electron plasma is created in the material, characterized by the electron plasma temperature $T_{e}$, and the plasma density $N_{e}$.

The ripples formation has been explained by Shimotsuma et al. [25] as the interference between the incident light field and the electric field of the electron plasma wave in material. An analytical expression of the ripples period has been proposed with the following form:

$$
\Lambda=\frac{2 \pi}{\sqrt{\frac{1}{T_{e}}\left(\frac{m_{e} \omega^{2}}{3 k_{B}}-\frac{e^{2} N_{e}}{3 \varepsilon_{0} k_{B}}\right)-k_{p h}^{2}}}
$$

were $m_{e}$ is electron mass, $k_{B}$ is Boltzmann constant, $\omega$ is angular frequency, and $k_{p h}$ is the wave vector of the incident light. From the above expression we can presume that the parameters of the electron plasma will have a very nonlinear impact on the ripples formation.

Therefore, when the laser fluence increases, the photoelectrons created by multiphoton photoionization process are more energetic and the electron plasma temperature increase. Following the dependence of the ripples period with the electron plasma temperature from relation (1) we can expect an increasing of $\Lambda$ with the laser energy, as experimentally observed.

On the other hand, the dependence of the ripples spacing with the scanning speed or equivalently with the number of pulses is hard to be interpreted. However, the fitting function from the figure 1 compared with the relation (1) can suggest a relation between the electron plasma's parameters and the number of pulses. Apparently, the increase of the number of pulses will modify the electrons temperature, or the density of the electron plasma, in a competitive manner, depending strongly on the way the radiation is absorbed in the material.

As shown by Reif et al. [26], from pulse to pulse the irradiated material will change into a "soft" state with different electronic properties, therefore, with different absorption properties. It is possible that increasing the number of pulses the effective dose of absorbed radiation will vary, inducing a nonlinear modification of electron plasma density or electron plasma temperature.

Even if the ripples formation is not completely understood and controlled yet, some applications of LIPSS can be already expected. Such periodical surface structuring technique can be used in fabrication of nanogratings, micropolarisers, or for increasing the effective sensitive surfaces of the microsensors.

In figure 4 we show the SEM image of a part of a large processed area (about $200 \times 500 \mu \mathrm{m}^{2}$ ) of a $\mathrm{ZnO}$ film. The structure was created by multiple scans parallel to the polarisation direction. The processing parameters were the following: scanning speed $0.1 \mathrm{~mm} / \mathrm{s}$, scanning offset 0.5 $\mu \mathrm{m}$, laser fluence $0.34 \mathrm{~J} / \mathrm{cm} 2$. The laser beam diameter 
defined at $1 / \mathrm{e}^{2}$ from the maximum intensity is estimated to be about $3 \mu \mathrm{m}$ in the focal spot. However, since the laser intensity is kept at the ablation threshold, the width of a scanned line is about $500 \mathrm{~nm}$.

The created structure has the period of about $170 \mathrm{~nm}$ and about $50 \mathrm{~nm}$ spacing between ripples. The ripples from parallel scans are coherently matched to each other forming continuous lines over a wide area, perpendicular to the scanning direction.

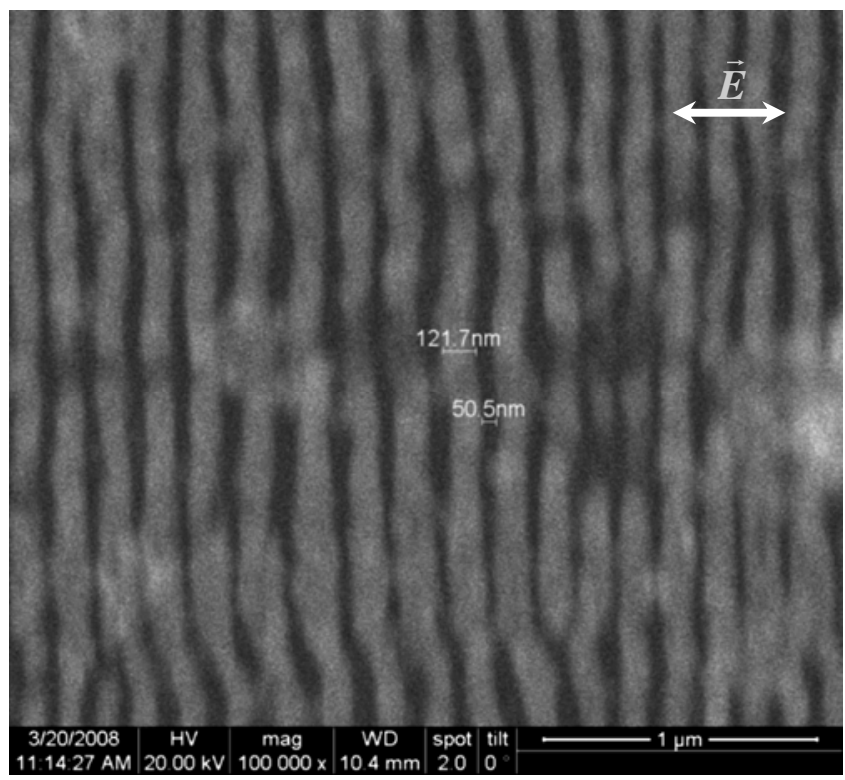

Fig. 3 Nanograting obtained on $\mathrm{ZnO}$ film by multiple scan parallel to the polarisation direction. Scanning speed is 0.1 $\mathrm{mm} / \mathrm{s}$, scanning offset $0.5 \mu \mathrm{m}$, and laser fluence 0.34 $\mathrm{J} / \mathrm{cm}^{2}$. The laser beam diameter is $3 \mu \mathrm{m}$ and the width of each scan line is about $500 \mathrm{~nm}$.

\section{Conclusions}

Periodically structures perpendicular to the laser polarisation were created in $\mathrm{ZnO}$ films under laser irradiation above the ablation threshold. The period of the laser induced structures is in the range of 130 to $200 \mathrm{~nm}$ depending on laser energy and scanning speed. The depth of the created grooves is from 50 to $140 \mathrm{~nm}$, depending on the laser fluence and the scanning speed, and is comparable with the thickness of the film. The width of the grooves is about $50 \mathrm{~nm}$, suggesting a high aspect ration from 1:1 up to $3: 1$. If proper experimental conditions are chosen, continuous structures over a few microns without cracks and only a few bifurcations can be done. A nanograting with period of $170 \mathrm{~nm}$, more than $100 \mathrm{~nm}$ depth and total surface of $200 \times 500 \mu \mathrm{m}^{2}$ was created on $\mathrm{ZnO}$. The sample was multiple scanned along the polarisation direction in order to obtain periodic structures over a large area of few hundreds of $\mu \mathrm{m}^{2}$.

\section{Acknowledgments}

This work was supported by the National Research Programe CEEX-ET 5848.

\section{References}

[1] M. Birmbaum: J.Appl. Phys., 36, (1965) 3688.
[2] N. Mansour, G. Reali, P. Aiello, and M.J. Soileau: "Laser Induced Damage in Optical Materials", ed. by By Harold E. Bennett, Arthur H. Guenther, David Milam, Brian E. Newman, (ASTM International, 1986) 137.

[3] R. Wagner and J. Gottmann: J. Phys. Conf. Ser. 59 (2007) 333.

[4] P. A. Temple and M. J. Soileau: IEEE J. Quant. Elec. QE-17, (1981) 2067.

[5] J. E. Sipe, J. F. Young, J. S. Preston, and H. M. van Driel: Phys. Rev. B 27, (1983) 1141.

[6] Z. Guosheng, P. M. Fauchet, and A. E. Siegman: Phys. Rev. B, 26, (1982) 5366.

[7] T. Tomita, K. Kinoshima, T. Murai, Y. Fukumori, S.Matsuo, S. Hashimoto: J. Laser Micro/Nanoengineering, 2, (2007) 141.

[8] Q. Z. Zhao, S. Malzer, and L. J. Wang: Opt. Lett., 32, (2007), 1932.

[9] T. Q. Jia, H. X. Chen, M. Huang, F. L. Zhao, J. R. Qiu, R. X. Li, Z. Z. Xu, X. K. He, J. Zhang, H. Kuroda, Phys. Rev. B 72, 125429 (2005).

[10] M. Couillard, A. Borowiec, H. K. Haugen, and J. S. Preston, E. M. Griswold, G. A. Botton: J. Appl. Phys., 101, (2007), 033519.

[11]F. Costache, M. Heinik, J. Reif, Appl. Surf. Sci., 186, (2002) 352.

[12] O. Varlamova, F. Costache, M. Ratzke and J. Reif: Appl. Surf. Sci., 253, (2007) 7932.

[13] R. Wagner, J. Gottmann, A. Horn and E. W. Kreutz: Appl. Surf. Sci. 252, (2006) 8576.

[14]B. K. Nayak, M. C. Gupta and K. W. Kolasinski, Appl. Surf. Sci., 253, (2007), 6580.

[15] M. Guillermin, F. Garrelie, N. Sanner, E. Audouard, and H. Soder: Appl. Surf. Sci. 253, (2007), 8075.

[16] M. Tang, H. Zhang, T. H. Her, Nanotechnology, 18, (2007), 485304.

[17] M. Gedvilas, G. Račiukaitis, K. Regelskis and P. Gečys: Journal of Laser Micro/Nanoengineering, 3, (2008) 58.

[18] J. Gottmann, D. Wortmann, and N. Brandt: Proc. SPIE, $6879,(2008) 68790 \mathrm{~N}$.

[19] J. C. Bernède, Y. Berredjem, L. Cattin, and M. Morsli: Appl. Phys. Lett. 92, (2008) 083304.

[20] S. F. Chichibu, T. Onuma, M. Kubota, A. Uedono, T. Sota, A. Tsukazaki, A. Ohtomo, and M. Kawasaki : J. Appl. Phys. 99, (2006) 093505.

[21]R. Shimada, J. Xie, V. Avrutin, Ü. Özgür, and H. Morkoč: Appl. Phys. Lett. 92, (2008) 011127.

[22] M. Huang, F. L. Zhao, T. Q. Jia, Y. Cheng, N. S. Xu, Z. Z. Xu: Nanotechnology, 18, (2007), 505301.

[23] X. D. Guo R. X. Li, Y. Hang, Z. Z. Xu, B. K. Yu, H. L. Ma, X.W. Sun: Materials Lett. 61 (2007) 4583.

[24] X. D. Guo, R. X. Li, Y. Hang, Z. Z. Xu, B. K. Yu, H. L. Ma, B. Lu and X.W. Sun: Materials Lett., 62, (2008) 1769.

[25] Y. Shimotsuma, P. G. Kazansky, L. Qiu, and K. Hirao: Phys. Rev. Lett. , 91, (2003) 247405.

[26] J. Reif, F. Costache, S. Eckert: J. Phys.: Conf. Ser. 59 (2007) 1-4.

(Received: June 16, 2008, Accepted: January 14, 2009) 